\title{
Robustness of Tukey's Control Chart in Detecting a Changes of Parameter of Skew Distributions
}

\author{
Saowanit Sukparungsee
}

\begin{abstract}
The observations are not always identically and independently distributed (i.i.d.) in continuous manufacturing which most observations is skewed away normal distribution. In this research aim to study the performance of Tukey's control chart for detecting a change in parameter when observation are from skew distributions such as exponential and Laplace distributions. Also, the performance of Tukey's control chart is compared with Shewhart and Exponentially Weighted Moving Average (EWMA) charts. The Average Run Length is commonly measured the performance of control chart. The ARLO is usually used when the process is in-control (sufficient large) and the ARL1 is correctly signaled to be outof-control (minimum). The Tukey's control chart is superior to other charts for large shift; however, the EWMA performs better for small to moderate shifts. The Tukey's control chart is very easy to setup the control limits and use a simple statistical concept.
\end{abstract}

Index Terms-Average Run Length, Skew Distributions, EWMA, Tukey's Control Chart, Robustness.

\section{INTRODUCTION}

Statistical Process Control (SPC) charts are widely used for monitoring, measuring, controlling and improving quality of production in many areas of application, for example, in industry and manufacturing, finance and economics, epidemiology and health care, environmental sciences and other fields. Control charts are usually designed and evaluated under the assumption that the observations from a process are independent and identically distributed (i.i.d.) and from a normal distribution. In real applications, there are many situations in which the process data come from a non-normal distribution, for example, an Exponential, Laplace, Student-t or Gamma distribution (see, e.g., Borror et al. [1]; Stoumbos and Reynolds [2]; Mititelu et al. [3]). Processes with data from a non-normal distribution need to be monitored by appropriate control charts.

Recently, many types of control charts are proposed which an appropriate control chart must be selected under many assumptions and several factors. Specially, for this kind of process monitoring, individual control charts take only one sample to measure due to economic issue for a company. Consequently, Tukey's control chart has been popular used for individual process which Alemi (2004) who was first proposed. Torng and Lee (2008) [4] and Torng et al. (2009) [5] have been investigated the average

Manuscript received May 5, 2012; revised June 11, 2012.

S. Sukparungsee is with the Department of Applied Statistics, Faculty of Applied Science, King Mongkut's University of Technology North Bangkok, Bangkok 10800, Thailand (tel.: +662-6538802; fax: + 6626538802; e-mail: swns@kmutnb.ac.th). run length of Tukey's control chart. There are many advantage of using Tukey's control chart which it is easy to use and simple control limits setup. It can be used with not only non-Normal observations but also when distribution of process is unknown. Furthermore, Tukey's control chart does not sensitive to unusual data such as an outlier.

Consequently, this paper aim to study the performance of Tukey's control chart robust to the skew distribution processes such exponential and Laplace distributions. They are usually represented as lifetime of products and growth rate of a company, respectively.

\section{CONTROl Charts AND Properties}

Let $X_{t}, \mathrm{t}=1,2, \ldots$ be a sequence of independent identically distributed (i.i.d.) random variables with a distribution $F(x, \alpha)$, where $\alpha$ is a parameter. It is usually assumed that under some "standard" (or "incontrol") conditions the parameter has a known in-control value $\alpha=\alpha_{0}$. Then at some unknown time $v$, which is called the change-point time $(v<\infty)$, the parameter $\alpha$ could be changed to an "out-of-control" value $\alpha \neq \alpha_{0}$.

\section{A. Tukey's Control Chart with Symmetrical Control Limits}

In 2004, Alemi [6] who first presented the Tukey's control chart which applied the principle of Box plot to obtain the control limits. The control limits of Tukey's control chart are proposed by Torng and Lee (2008) as follows:

$$
\begin{gathered}
U C L=F^{-1}(0.75)+L \times I Q R \\
\text { and } \quad L C L=F^{-1}(0.25)-L \times I Q R
\end{gathered}
$$

where UCL and LCL are upper and lower control limits, respectively. $F^{-1}(0.75)$ and $F^{-1}(0.25)$ are the third quartile $\left(\mathrm{Q}_{3}\right)$ and first quartile $\left(\mathrm{Q}_{1}\right)$ and IQR is the InterQuartile Range $\left(\mathrm{IQR}=\mathrm{Q}_{3}-\mathrm{Q}_{1}\right)$. The value of $\mathrm{L}$ is a coefficient of control limit which this value of $\mathrm{L}$ is usually set as 1.5 for the case of a normal distribution assumption (Ryan (2000)).

\section{B. Exponentially Weighted Moving Average (EWMA) control chart.}

The Exponentially Weighted Moving Average (EWMA) statistics for the discrete time case has the following form

$$
Z_{t}=\lambda\left(X_{t}-\mu_{0}\right)+(1-\lambda) Z_{t-1}, \quad 0<\lambda<1,
$$

where $\lambda$ is a weighting factor for previous observations. 
The target in-control parameter $\alpha_{0}$ is supposed to be steady and the initial value $Z_{0}$ is usually chosen to be the process in-control parameter, i.e. $Z_{0}=\alpha_{0}$. If the observations $X_{t}$ are independent random variables with variance $\sigma^{2}$, then the variance of EWMA statistics $Z_{t}$ is

$$
\sigma_{X_{t}}^{2}=\sigma^{2}\left(\frac{\lambda}{2-\lambda}\right)\left[1-(1-\lambda)^{2 t}\right], \mathrm{t}=1,2, \ldots
$$

Since $0<1-\lambda<1$, we have that $(1-\lambda)^{2 t} \rightarrow 0$ as $t \rightarrow \infty$, and therefore the asymptotic value of the variance is

$$
\sigma_{X_{t}}^{2}=\sigma^{2}\left(\frac{\lambda}{2-\lambda}\right) .
$$

The standard deviation used in the limits is usually the asymptotic value. Using the expression in Equation (3), the control limits of the EWMA chart is the following:

$$
\begin{array}{r}
U C L=H_{U}=\alpha_{0}+L \sigma \sqrt{\frac{\lambda}{2-\lambda}} \\
\text { and } L C L=H_{L}=\alpha_{0}-L \sigma \sqrt{\frac{\lambda}{2-\lambda}},
\end{array}
$$

where $L$ is a constant to be chosen. The process will be declared to be in an out-of-control state when $X_{t}>H_{U}$ or $X_{t}<H_{L}$ (see Sukparungsee and Novikov [7])

\section{Shewhart Control Chart}

Let $X_{1}, X_{2}, \ldots, X_{t}$ be independent and identically random variables (observations).

The level A and -A are called the upper control limit (UCL) and the lower control limit (LCL). Traditionally,

$$
\begin{aligned}
U C L & =A=\alpha_{0}+L \frac{\sigma}{\sqrt{n}} \\
\text { and } L C L & =-A=\alpha_{0}-L \frac{\sigma}{\sqrt{n}},
\end{aligned}
$$

where $\mathrm{L}$ is constant (usually $\mathrm{L}=3$ for the case of normal distribution)
As stated above, one of the popularly important characteristics for SPC charts is Average Run Length (ARL) - the expectation of an alarm time $(\tau)$ is taken to signal (wrongly) about a possible change. Ideally, an acceptable ARL of in-control process should be enough large and a small ARL when the process is out-of-control, so-called Average of Delay time (AD) - the expectation of delay for true alarm time.

In the case of Tukey's and Shewhart control charts, ARL and $\mathrm{AD}$ can be easily calculated in terms of the error probabilities- the probability of type $\mathrm{I}$ error $\left(\mathrm{P}_{\mathrm{I}}\right)$ (the test rejects "true" $\mathrm{H}_{0}$ :signal occurs when a point falls outside the control limit when there is no real change in parameter) and the probability of type II error $\left(\mathrm{P}_{\mathrm{II}}\right)$ (the test rejects "true" $\mathrm{H}_{1}$ : no signal occurs when a point falls outside the control limit when there is a real change in parameter). The corresponding formulas are

$$
A R L_{0}=\frac{1}{P_{I}}
$$

and

$$
A R L_{1}=\frac{1}{1-P_{I I}}
$$

where

$$
P_{I}=1-\int_{L C L}^{U C L} f(X) d x
$$
$P_{I I}=1-\int_{L C L-\delta \sigma}^{U C L-\delta \sigma} f(X) d x, f(x)$ be the probability density function (pdf) of population, and $\delta$ is the shift size coefficient; $\delta=\alpha-\alpha_{0} / \sigma$.

\section{COMPARISON AND NUMERICAL RESUlTS}

The selected skew population in this study is exponential and laplace (double exponential) distributions which incontrol parameter of exponential is $1,1 / 3$ and $1 / 5$ and shift size $(\delta)$ is $0.01,0.05,0.1,0.5,1,1.5,2,3,4,5$. For the

\begin{tabular}{|c|c|c|c|c|c|c|c|}
\hline \multirow[t]{2}{*}{$\alpha_{0}$} & \multirow[t]{2}{*}{$\delta$} & \multicolumn{3}{|c|}{$\mathrm{ARL}_{0}=100$} & \multicolumn{3}{|c|}{$\mathrm{ARL}_{0}=370.4$} \\
\hline & & Tukey & Shewhart & $\begin{array}{c}\text { EWMA } \\
\lambda=0.05\end{array}$ & Tukey & Shewhart & $\begin{array}{c}\text { EWMA } \\
\lambda=0.05\end{array}$ \\
\hline \multirow[t]{4}{*}{1} & 0.05 & 95.914 & 79.551 & $70.064^{*}$ & 129.571 & 277.764 & $214.345^{*}$ \\
\hline & 0.1 & 91.267 & 64.937 & $51.053^{*}$ & 123.183 & 216.993 & $140.147^{*}$ \\
\hline & 0.5 & 61.194 & 21.660 & $13.699^{*}$ & 82.598 & 51.053 & $24.521^{*}$ \\
\hline & 1 & 37.107 & 10.003 & $7.112^{*}$ & 50.105 & 18.929 & $11.299^{*}$ \\
\hline
\end{tabular}
latter, in-control parameter is Laplace $(2,1)$ and shift size $(\delta)$ is $0.1,0.2,0.3,0.5,1,2,3$. The performance of Tukey's control chart is compared with Shewhart and EWMA charts when given ARL $=100$ and 370.4. Table I and II show the comparison of the performance of control charts when observations are exponential and Laplace distributed, respectively.

TABLE I: COMPARISON OF THE PERFORMANCE OF CONTROL CHARTS FOR THE CASE OF EXPONENTIAL DistRIBUTION 


\begin{tabular}{|c|c|c|c|c|c|c|c|}
\hline & 2 & 13.648 & 4.593 & $3.964^{*}$ & 18.431 & 7.110 & $5.767^{*}$ \\
\hline & 3 & 5.021 & 3.128 & $2.949^{*}$ & 6.780 & 4.353 & $4.068^{*}$ \\
\hline & 4 & $1.847^{*}$ & 2.494 & 2.490 & $2.494^{*}$ & 3.243 & 3.246 \\
\hline & 5 & $1^{*}$ & 2.175 & 2.150 & $1^{*}$ & 2.696 & 2.805 \\
\hline \multirow[t]{8}{*}{$1 / 3$} & 0.05 & 95.465 & 92.066 & $90.617^{*}$ & 352.187 & 333.407 & 310.758 \\
\hline & 0.1 & 90.854 & 84.956 & $83.946^{*}$ & 335.025 & 308.628 & $263.521^{*}$ \\
\hline & 0.5 & 60.907 & 50.511 & $49.379^{*}$ & 224.667 & 159.269 & $105.053^{\prime \prime}$ \\
\hline & 1 & 36.941 & 34.280 & $33.144^{*}$ & 136.146 & 85.559 & $56.342^{*}$ \\
\hline & 2 & 13.587 & 15.612 & $20.462^{*}$ & 50.065 & 35.005 & $29.092^{*}$ \\
\hline & 3 & $4.999^{*}$ & 9.983 & 14.817 & $18.432^{*}$ & 19.325 & 20.120 \\
\hline & 4 & $1.839^{*}$ & 7.155 & 11.849 & $6.774^{*}$ & 12.733 & 15.720 \\
\hline & 5 & $1^{*}$ & 5.638 & 9.974 & $2.493^{*}$ & 9.132 & 12.881 \\
\hline \multirow[t]{8}{*}{$1 / 5$} & 0.05 & $94.917^{*}$ & 95.197 & 95.541 & 352.572 & 348.666 & $334.028^{*}$ \\
\hline & 0.1 & $90.278^{*}$ & 91.378 & 90.683 & 334.048 & 329.791 & $298.463^{\prime \prime}$ \\
\hline & 0.5 & $60.508^{*}$ & 65.834 & 64.234 & 224.709 & 220.020 & 157.628 \\
\hline & 1 & $36.713^{*}$ & 45.451 & 47.130 & 136.325 & 138.821 & $91.412^{*}$ \\
\hline & 2 & $13.646^{*}$ & 27.003 & 31.419 & 50.144 & 68.402 & $49.127^{*}$ \\
\hline & 3 & $4.965^{*}$ & 17.678 & 23.750 & $18.446^{*}$ & 40.355 & 34.034 \\
\hline & 4 & $1.827^{*}$ & 12.760 & 19.235 & $6.785^{*}$ & 26.567 & 26.505 \\
\hline & 5 & $1^{*}$ & 10.065 & 16.374 & $2.497^{*}$ & 19.586 & 21.524 \\
\hline
\end{tabular}

* give minimum $\mathrm{ARL}_{1}$

TABLE II: COMPARISON OF THE PERFORMANCE OF CONTROL CHARTS FOR THE CASE OF LAPLACE DISTRIBUTION

\begin{tabular}{|c|c|c|c|c|c|c|c|}
\hline \multirow[t]{2}{*}{$\alpha_{0}$} & \multirow[t]{2}{*}{$\delta$} & \multicolumn{3}{|c|}{$\mathrm{ARL}_{0}=100$} & \multicolumn{3}{|c|}{$\mathrm{ARL}_{0}=370.4$} \\
\hline & & Tukey & Shewhart & $\begin{array}{c}\text { EWMA } \\
\lambda=0.05\end{array}$ & Tukey & Shewhart & $\begin{array}{c}\text { EWMA } \\
\lambda=0.05\end{array}$ \\
\hline & 0.1 & 86.626 & 99.372 & $69.146^{*}$ & 322.806 & 366.560 & $169.26^{*}$ \\
\hline & 0.3 & 65.899 & 91.972 & $43.45 * 1$ & 242.509 & 339.133 & $70.773 *$ \\
\hline & 0.5 & 49.346 & 79.620 & $32.432 *$ & 185.115 & 293.492 & $45.592 *$ \\
\hline & 1.0 & 24.127 & 46.085 & $20.650 *$ & 90.826 & 169.733 & $25.679 *$ \\
\hline & 2.0 & $5.998^{*}$ & 11.824 & 12.342 & 21.994 & 43.618 & $14.522 *$ \\
\hline & 3.0 & $1.555^{*}$ & 2.884 & 8.941 & $5.433^{*}$ & 10.633 & 10.321 \\
\hline & 4.0 & $1 *$ & 1.213 & 7.075 & $1.481 *$ & 2.590 & 8.104 \\
\hline
\end{tabular}

\section{CONCLUSION}

We have compared the performance of Tukey's control chart with Shewhart and EWMA charts when observations are from exponential and Laplace distributions. The ARL of Tukey's chart is calculated by the Equation (4) while Shewhart and EWMA approximate the ARL by Monte Carlo simulation. We found that the performance of EWMA is superior to other charts for small to moderate shifts when in-control parameter of exponential distribution is 1 and $1 / 3$ and Tukey's control chart is good to detect large shifts for both case of $\mathrm{ARL}_{0}=100$ and 370.4. When in-control parameter is $1 / 5$ the performance of Tukey's control chart shows the best performance for all shift sizes with both case of $\mathrm{ARL}_{0}=100$ and 370.4. Tukey's control chart is also robust to Laplace distribution process and the numerical results are good agreement with the former case study.

\section{ACKNOWLEDGEMENTS}

The author would like to give special thanks to, King Mongkut's University of Technology, North Bangkok, 
Thailand for a supporting research grant.

\section{REFERENCES}

[1] C. M. Borror, D. C. Montgomery, and G. C. Runger, "Robustness of the EWMA Control Chart to Non-normality," Journal of Quality Technology, vol. 31, no. 3, pp. 309-316, 1999.

[2] Z. G. Stoumbos and M. R. Reynolds, "Robustness to non-normality and autocorrelation of individuals control charts for monitoring the process mean and variance," Journal of Statistical Computation and Simulation, vol. 66, pp. 145-187, 2000.

[3] G. Mititelu, Y. Areepong, S. Sukpakrungsee, and A. Novikov, "Explicit Analytical Solutions for the Average Run Length of CUSUM and EWMA Charts," East-West Journal of Mathematics, Special Volume, pp. 253-265, 2010.
[4] C. C. Torng and P. H. Lee, "ARL Performance of the Tukey's Control Chart," Communications in Statistics: Simulation and Computation, vol. 38, no. 3, pp. 541-557, 2008.

[5] C. C. Torng and P. H. Lee, "The Performance of Double Sampling $\bar{x}$ Control Charts Under Non-Normality," Communications in Statistics: Simulation and Computation, vol. 37, no. 9, pp. 1904-1913, 2008.

[6] F. Alemi, “Tukey's Control Chart," Quality Management in Health Care, vol. 13, no. 4, pp. 216-221, 2004.

[7] S. Sukparungsee and A. A. Novikov, "On EWMA Procedure for Detection of a Change in Observations via Martingale Approach," KMITL Science Journal: An International Journal of Science and Applied Science, vol. 6, no. 2b, pp. 373-380, 2006. 